\title{
Specific Effectiveness of Acupuncture and Kampo Medicine on Mice with Depression
}

\author{
Ko Nishimura ${ }^{1,2 *}$, Jun Kawanokuchi ${ }^{1}$, Teruhisa Yamamoto ${ }^{2}$, Ken Takagi ${ }^{1,2}$, Kaito Mizuno ${ }^{1,2}$, Torao Ishida $^{1}$ and \\ Atsushi Ishige ${ }^{3}$
}

${ }^{1}$ Institute of Traditional Chinese Medicine, Suzuka University of Medical Science Suzuka, Japan

${ }^{2}$ Department of Acupuncture and Moxibustion, Suzuka University of Medical Science Suzuka, Japan

${ }^{3}$ Yokohama College of pharmacy Yokohama, Japan

Submission: December 17, 2019; Published: January 13, 2020

*Corresponding author: Ko Nishimura, Institute of Traditional Chinese Medicine, Suzuka University of Medical Science, Japan

\begin{abstract}
Although early recovery and prevention of depression are important in modern society, antidepressant, that is often the primary pharmacotherapy, produces unwanted adverse effects. We investigated preventive and therapeutic effects, and additionally difference of monotherapy and combined therapy using major traditional medicines, i.e. Kampo (Japanese traditional herbal medicine, saikokaryukotsuboreito) and acupuncture (GV20 and Ex-HN3 as acupoints), which have much less side effects than Western medicine, compared with imipramine measuring immobility time brought by forced swimming (FS) stress. Furthermore, we analyzed its mechanisms according to changes in neurotrophic factors by molecular biology/biochemical method. All the intervention groups in the preventive evaluation and the therapeutic groups in the therapeutic evaluation did not worsen immobility time, and improved prolonged immobility time induced by FS stress respectively. However, there was no significant difference in the time between either the intervention groups or the therapeutic groups. BDNF and NT-3 in acupuncture and combined groups were higher than those in FS group both in the preventive and the therapeutic groups. NGF in the combined group was as same as FS group, and higher than that in acupuncture, Kampo, and imipramine groups in both the preventive and the therapeutic groups. These findings suggest that acupuncture and Kampo improve depression through the regulation of neurotrophic factors, of which mechanism is different from imipramine, and that combined therapy has specific anti-depressive mechanism that does not exert in acupuncture or Kampo alone. We need to utilize more depression rating scales to evaluate the effect of combined therapy more precisely.
\end{abstract}

Keywords: Depression; Kampo medicine (Japanese traditional herbal medicine); Acupuncture; Complementary and alternative medicine; Forced swimming stress; Neurotrophic factor

\section{Introduction}

Depression is a psychiatric disorder classified as mood disorder [1]. Since the prevalence of depression is high, early recovery and prevention of onset are important in modern society. The treatment of depression includes pharmacotherapy, electroconvulsive therapy, and psychotherapy. Pharmacotherapy with antidepressant is often the primary treatment for depression. However, antidepressants may produce unwanted adverse effects. Consequently, patients with depression should often take some additional drugs to reduce the side actions [2].

Therefore, some patients are seeking complementary and alternative medicine for depression to avoid polypharmacy and take treatment that is more effective. In fact, there are several reports about effectiveness of complementary and alternative treatment such as acupuncture or traditional herbal medicine [3-7]. Acupuncture and traditional herbal medicine have been utilized as a treatment for physical and mental disorders in traditional Oriental medicine for a long time $[8,9]$. The role of these treatments is to regulate energy imbalance in the body. Because of their almost no side effects, even in Western countries, practitioners have been focusing on them as a potential new approach [10]. Unfortunately, they have been usually used as monotherapy respectively. Although it has been said that acupuncture and traditional herbal medicine exert much power working together, almost patients actually take either acupuncture or traditional herbal medicine in clinical practice.

There are various reports on mechanisms of depression. However, clear conclusions have not yet been obtained. Depression has been considered to involve decreases in intracerebral levels of monoamines for about 60 years [11,12]. This hypothesis cannot be denied, because tricyclic antidepressants indeed have 
partial effect for depression. On the other hand, it has been found that neurogenesis is related to depression, and brain derived neurotrophic factor (BDNF) is one of the important factors for neurogenesis [13-15]. In 2000, Berman RM, et al. [16] reported that ketamine has immediate effect for depression, and BDNF was found to be importantly related to the effect of ketamine [17]. Given this situation, neural plasticity seems to have been gaining attention and settling as the hypothesis of depression onset [1820].

In this study, Kampo medicine, which is Japanese traditional herbal medicine, and acupuncture, both of which are popular traditional Oriental medicine with fewer side effects than Western medicine, were performed simultaneously on depression model mice to compare the effect between monotherapy and combined therapy. Furthermore, its mechanisms were analyzed by molecular biology/biochemical method. This seems to be the first investigation about the effect of acupuncture and Kampo combined therapy for depression as far as we know.

\section{Experimental Procedures}

We investigated the antidepressant effect of acupuncture, Oriental herbal medicine, and Western medicine using depression animal model. Moreover, we focused on both the preventive and therapeutic potency. All protocols were approved by the Animal Experiment Ethical Review Committee of Suzuka University of Medical Science (authorization number: 189).

\section{Experimental animals}

We used 6-week-old male ICR mice as depression model. They were purchased from CLEA Japan, Inc. (Tokyo, Japan), and maintained under a room temperature of $22 \pm 3^{\circ} \mathrm{C}, 60 \%$ humidity, and a 12-h light/dark cycle. They took animal feed (CE-2; CLEA Japan, Inc.) and water as free access. We acclimatized them by handling for one week before the start of the experiment.

\section{Production of depression in mice}

We created depression model mice using the forced swimming (FS) procedure described by Porsolt, et al. [21]. ICR mice were placed in a plastic beaker (diameter, $15 \mathrm{~cm}$; height, $22 \mathrm{~cm}$ ) containing freshwater at $25 \pm 2^{\circ} \mathrm{C}$ to a depth of body height $+5 \mathrm{~cm}$ for $6 \min 5$ days a week for 2 weeks.

\section{Procedure of acupuncture and drug administration}

We mainly examined if there is difference in the antidepressant effect between the acupunctural monotherapy, Kampo monotherapy, and combined therapy of acupuncture and Kampo. Initially, the depression model mice were divided into two groups, i.e. group for evaluation of the preventive effect, and group for evaluation of the therapeutic effect. Then, the two groups were additionally classified into five groups respectively, i.e. just forced swimming stress group with no treatment (FS group), acupuncture group (FS+AP group), Kampo extract administration group (FS+
Kampo group), combined therapy with both Kampo extract administration and acupuncture group (FS+AP+ Kampo group), and imipramine administration group (FS+ Imipramine group). Furthermore, we set ICR mice with neither FS nor treatment as control group. Each group had eight subjects respectively. In the groups for evaluation of the preventive effect, we conducted forced swimming and acupuncture and/or drug treatment simultaneously.

In the acupuncture procedure, we used stainless steel needles (diameter, $0.25 \mathrm{~mm}$; length, $15 \mathrm{~mm}$, Acupuncture Needle D-Type) which were obtained from SEIRIN Co., Ltd., Shizuoka, Japan. The original needle is longer than $15 \mathrm{~mm}$, because it has a handle. We modified to make the handle cut out in order not to be removed by the mice. We selected "Bai-Hui" (DV20) and "Yintáng" (Ex-HN3) as acupuncture points according to previous reports that showed the effectiveness of acupuncture for depression using these acupoints $[6,22,23]$. DV20 is typically utilized for neurological and psychiatric diseases such as stroke, headache, dizziness, and anxiety. Ex-HN3, which has function to calm the spirit, is typically used for insomnia, anxiety, and stress. At these acupoints, horizontal and inward simultaneous acupuncture stimuli were continued for $20 \mathrm{~min}$ with insertion to a depth of $5 \mathrm{~mm}$. During the acupuncture stimulation, the mouse was fixed with adhesive tape on its tail on an overturned cage. These acupuncture stimuli were performed for 5 days a week for 2 weeks. The control mice that did not receive stimulation with acupuncture were just fixed for the same duration as AP group.

In the pharmacotherapy, we utilized saikokaryukotsuboreito as Kampo medicine and imipramine as Western antidepressant based on previous reports that showed the usefulness of these drugs [24-26]. Saikokaryukotsuboreito was obtained as a powdered extract $(4.5 \mathrm{~g})$ made from a mixture of Bupleuri radix (5.0 g), Scutellariae radix (2.5g), Ginseng radix (2.5g), Pinelliae tuber (4.0g), Zingiberis rhizoma (1.0g), Zizyphi fructus (2.5g), Cinnamomi cortex (3.0g), Poria (3.0g), Ostreae testa (2.5g), and Fossilia Ossis Mastodi (2.5g) from Tsumura \& Co, Tokyo, Japan (lot number: 2160012010). Imipramine was obtained from Sigma, MO, USA. The dose of these drugs were set according to previous reports. Saikokaryukotsuboreito $(1 \mathrm{~g} / \mathrm{kg}$ body weight) and imipramine $(10 \mathrm{mg} / \mathrm{kg}$ body weight) were dissolved in saline and administered orally with a gastric tube 5 days a week for 2 weeks. The control mice that did not receive pharmacotherapy orally took normal saline as the same way as the therapeutic groups

\section{Measurement method}

The evaluation of depression was determined by immobility time in the forced swimming test described above [21]. ICR mice were placed in a plastic beaker (diameter, $15 \mathrm{~cm}$; height, $22 \mathrm{~cm}$ ) containing freshwater at $25 \pm 2{ }^{\circ} \mathrm{C}$ to a depth of body height $+5 \mathrm{~cm}$ for 6 min 5 days a week for 2 weeks. The action of mouse was recorded with a video camera, and immobility time for $5 \mathrm{~min}$ after excluding the first $1 \mathrm{~min}$ from the 6 min recording time was 
measured. The immobile state was defined as a state in which the mouse suspends most movements and performs only those movements of the limbs necessary to maintain balance. Body weight was also measured in relation to depression.

In the sample collection, mice were immediately anesthetized after a series of animal experiments. Their brains were immediately removed and stored at $-80{ }^{\circ} \mathrm{C}$. Right anterior cerebrum was used for molecular biological and biochemical analysis. Expressions of cerebral neurotrophic factors were determined by RT-PCR and ELISA. Total RNA was extracted from brain tissue using RNeasy Mini kit according to the manufacturer's protocol (Qiagen, Valencia, CA, USA). The amount of RNA was determined spectrophotometrically. cDNAs encoding mouse glyceraldehyde- 3-phosphate dehydrogenase (GAPDH), nerve growth factor (NGF), BDNF, neurotrophin-3 (NT-3), and neurotrophin-4/5 (NT-4/5) were evaluated as the study subjects. To quantify the expression, mRNAs of these neurotrophic factors were analyzed by real-time RT-PCR using Super Script III (Invitrogen, Carlsbad, CA, USA), Power Up SYBER Green Master Mix (Applied Biosystems, TX, USA) and the ABI Prism 7000 SDS (Applied Biosystems). Thermal cycling parameters for primer optimization were as follows: activation at $95^{\circ} \mathrm{C}$ for $10 \mathrm{~min}$ followed by 50 cycles $95^{\circ} \mathrm{C}$ for $15 \mathrm{sec}$ and $60^{\circ} \mathrm{C}$ for $1 \mathrm{~min}$. The mRNA level for each sample was normalized against GAPDH mRNA. Specific primers are shown in Table 1. Expressions of cerebral neurotrophic factors were assessed using MultiNeurotrophin Rapid Screening ELISA kit: Mouse according to the manufacturer's protocol (Biosenis,SA,Australia).

Table 1: Primer sets for real time PCR

\begin{tabular}{|c|c|c|}
\hline Molecule & Forward Primer & Reverse Primer \\
\hline GAPDH & ATGGGAGTTGCTGTTGAAGTCA & CCGAGGGCCCACTAAAGG \\
\hline NGF & GATCGGCGTACAGGCAGAAC & GCAGCCTTCCTTGGTGTAACC \\
\hline BDNF & CCAAAGGCCAACTGAAGCAGTA & GGCAAACTCCTTTGATCCAT \\
\hline NT-3 & TTCTGCCACGATCTTACAGG & GGTCATGTTGGATGGGAGGTATC \\
\hline
\end{tabular}

\section{Statistical analysis}

Statistical significance was assessed using one-way analysis of variance (ANOVA) with Tukey-Kramer post-hoc using PRISM

\section{Results}

\section{Physical and behavioral findings}

version 5.0 (GraphPad Software, La Jolla, CA, USA). $P<0.05$ was defined as significant.

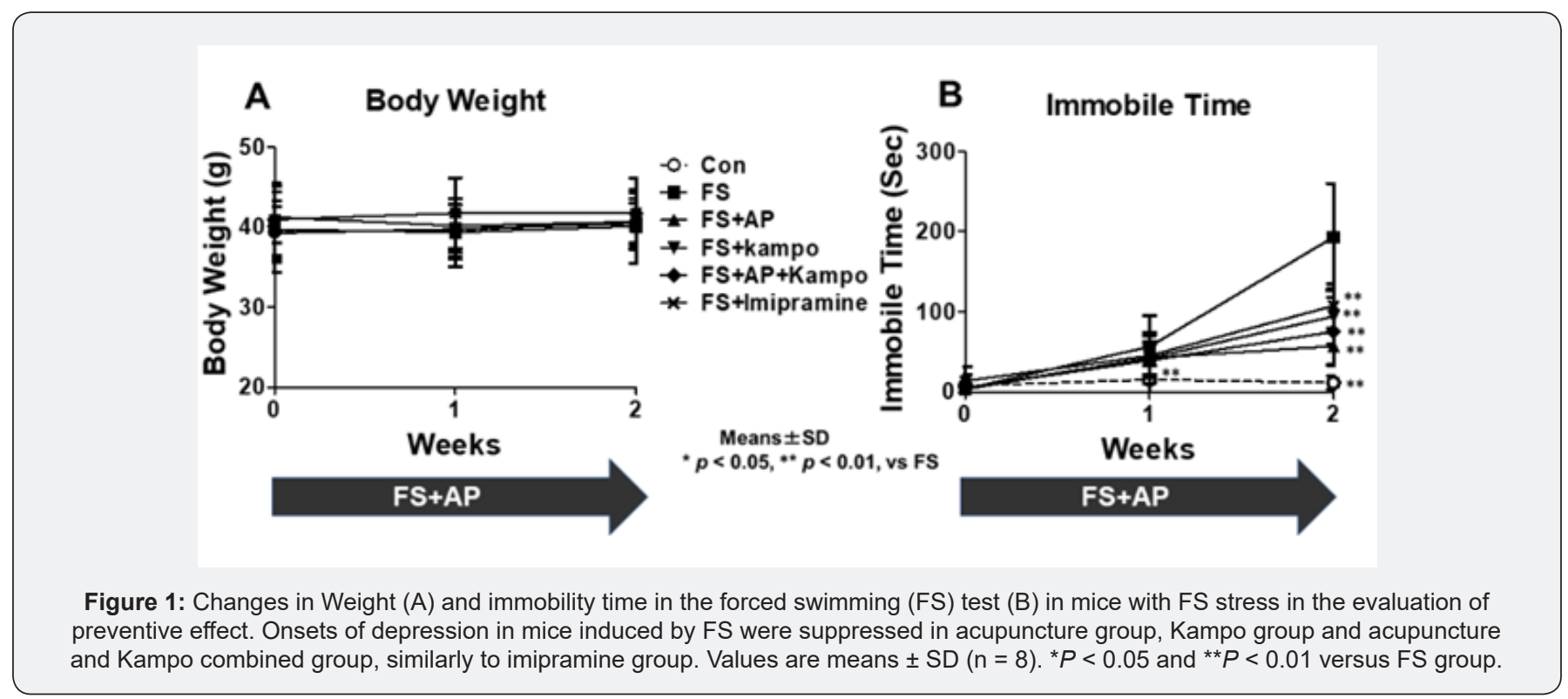

There was no significant difference in the body weight between any additional classified groups in either the group for evaluation of preventive effect or the group for evaluation of therapeutic effect (Figures 1A \& 2A). In the groups for evaluation of preventive effect, there was no significant difference in immobility time between
AP, Kampo, combined, and imipramine groups, and the time in FS group significantly increased compared with any other groups (Figure 1B). In the groups for evaluation of therapeutic effect, all the treatment groups had significant decrease in immobility time after treatment (Figure 2B). 

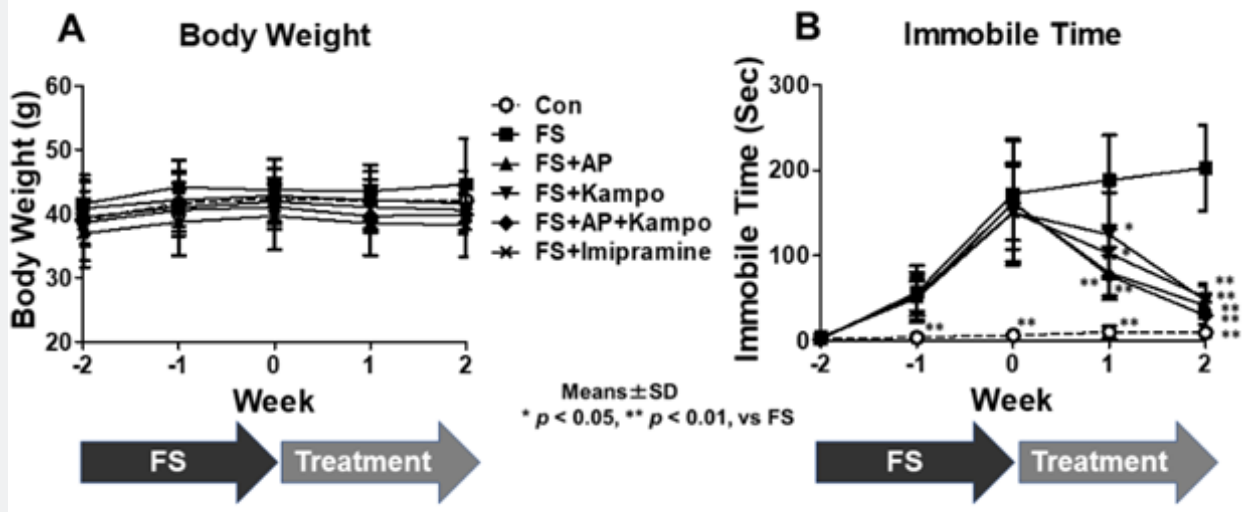

Figure 2: Changes in weight (A) and immobility time in the forced swimming test (B) in mice with FS stress in the evaluation of therapeutic effect. Acupuncture group, Kampo group and acupuncture and Kampo combined group showed therapeutic effects on depression mice induced by FS, similarly to imipramine group. Values are means $\pm \mathrm{SD}(\mathrm{n}=8)$. ${ }^{*} P<0.05$ and ${ }^{* *} P<0.01$ versus FS group.

\section{Molecular biology / biochemical findings}
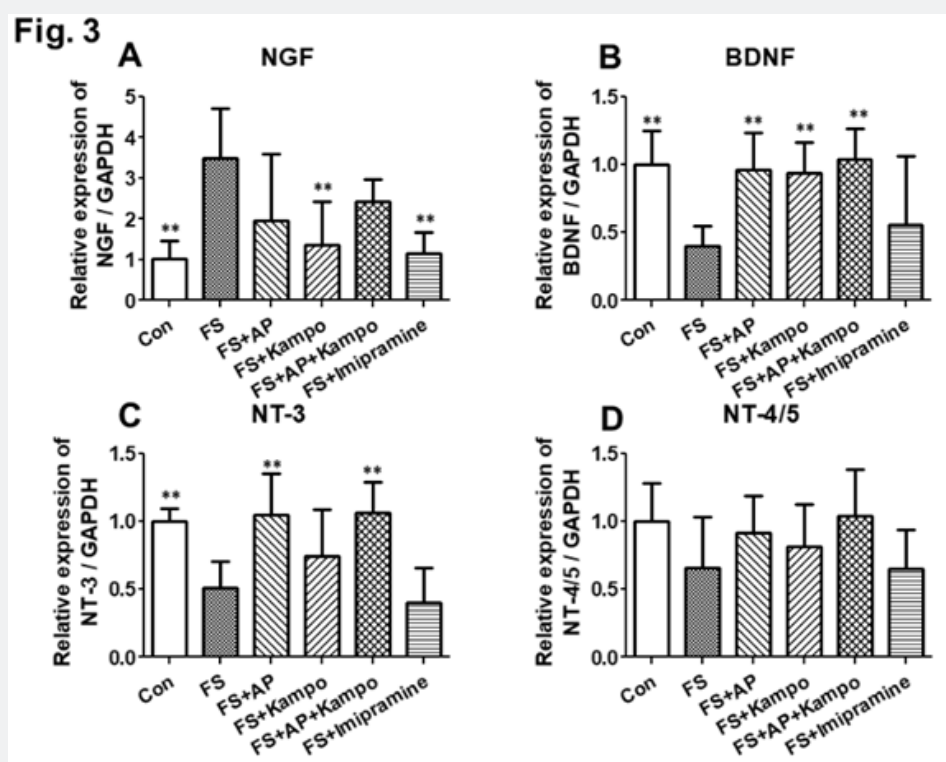

Figure 3: mRNA expressions of NGF (A), BDNF (B), NT-3 (C), NT-4/5 (D) in the mice brain in the evaluation of preventive effect. Results are expressed as relative gene expression levels of mRNA. Values are means $\pm \mathrm{SD}(\mathrm{n}=8)$. ${ }^{*} P<0.05$ and ${ }^{* \star} P<0.01$ versus FS group.

In the investigation of preventive effect, we had following results (Figures $3 \& 4$ ). mRNA expressions of NGF in control, FS+Kampo, and FS+Imipramine groups were significantly lower than that in FS group. Protein expressions of NGF in control, FS+AP, FS+Kampo, and FS+Imipramine groups were significantly lower than that in FS group, and the expression in combined group was as same as FS group. mRNA expressions of BDNF in control, $\mathrm{FS}+\mathrm{AP}$, $\mathrm{FS}+$ Kampo, and FS+AP+Kampo groups were significantly higher than that in FS group. Protein expressions of BDNF in control, $\mathrm{FS}+\mathrm{AP}$, and $\mathrm{FS}+\mathrm{AP}+\mathrm{Kampo}$ groups were significantly higher than that in FS group. mRNA expressions of NT-3 in control, FS+AP, and $\mathrm{FS}+\mathrm{AP}+\mathrm{Kampo}$ groups were significantly higher than that in
FS group. Protein expressions of NT-3 were observed in the same way as the mRNA expressions. There was no significant difference in mRNA expressions of NT-4/5 between any groups. Protein expressions of NT-4/5 in control and FS+AP+Kampo groups were significantly higher than that in FS group, and the expressions in AP, Kampo, and imipramine groups were as same as FS group.

In the investigation of therapeutic effect, we had following results (Figures 5,6). mRNA expressions of NGF in control, FS+AP, FS+Kampo, and FS+Imipramine groups were significantly lower than that in FS group, and the expression in combined group was as same as FS group. Protein expressions of NGF were similar to the 
mRNA expressions. mRNA expressions of BDNF in control, FS+AP, $\mathrm{FS}+$ Kampo, and $\mathrm{FS}+\mathrm{AP}+$ Kampo groups were significantly higher than that in FS group. Protein expressions of BDNF in control $\mathrm{FS}+\mathrm{AP}$, and $\mathrm{FS}+\mathrm{AP}+$ Kampo groups were significantly higher than that in FS group. mRNA expressions of NT-3 in control, FS+AP, $\mathrm{FS}+$ Kampo, and $\mathrm{FS}+\mathrm{AP}+$ Kampo groups were significantly higher than that in FS group. Protein expressions of NT-3 were observed in the same way as the mRNA expressions except in FS+Kampo group. There was no significant difference in mRNA expressions of NT- $4 / 5$ between any groups. Protein expression of NT-4/5 in only control group was significantly higher than that in FS group.
Fig. 4
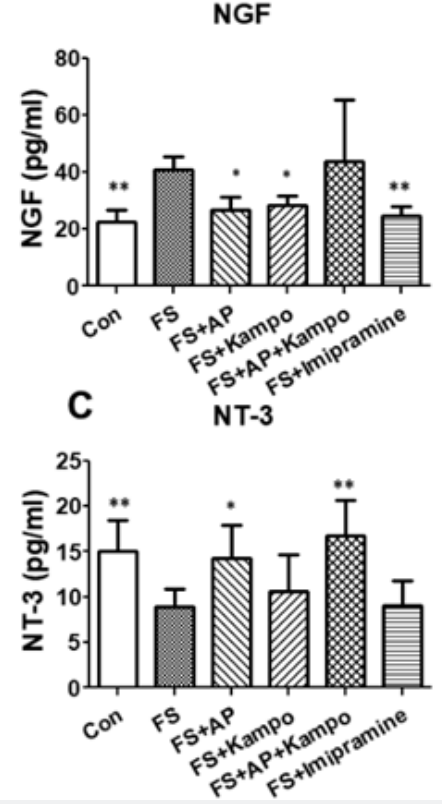

B BDNF
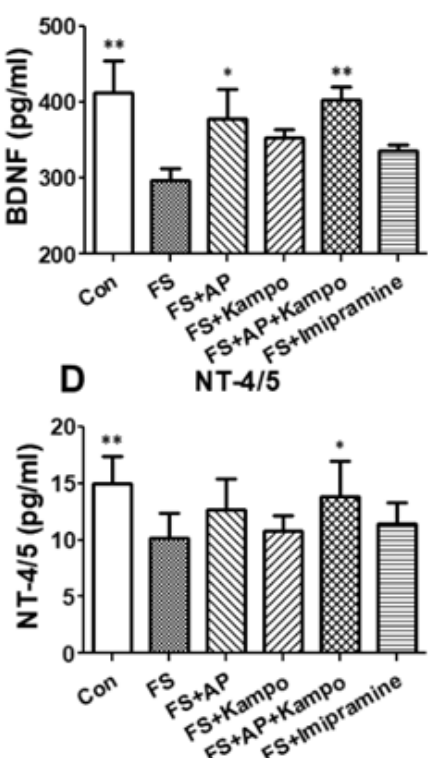

Figure 4: Expressions of NGF (A), BDNF (B), NT-3 (C), NT-4/5 (D) in the mice brain in the evaluation of preventive effect. Results are expressed as protein content measured by ELISA. Values are means \pm SD $(n=8)$. ${ }^{*} P<0.05$ and ${ }^{* *} P<0.01$ versus FS group.

\section{Fig. 5}
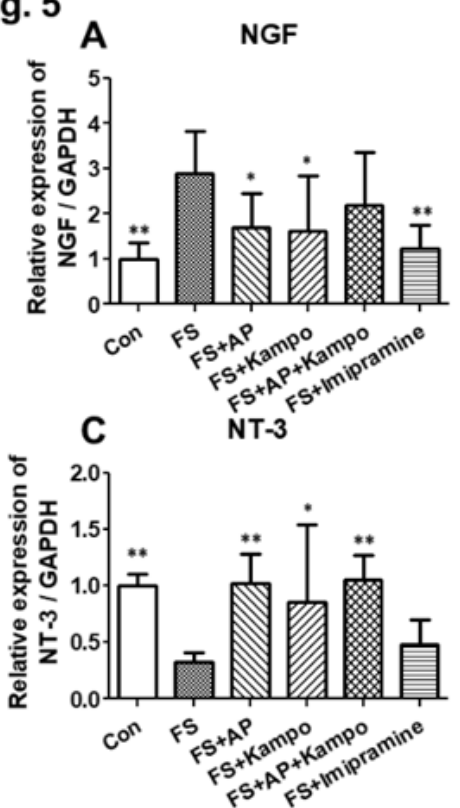

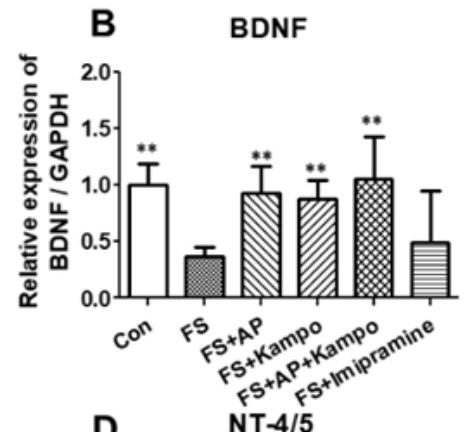

D NT-4/5

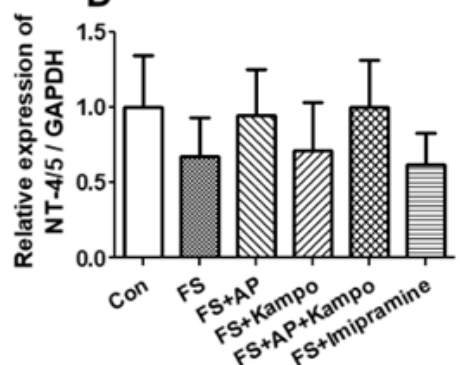

Figure 5: mRNA expressions of NGF (A), BDNF (B), NT-3 (C), NT-4/5 (D) in the mice brain in the evaluation of therapeutic effect. Results are expressed as relative gene expression levels of mRNA. Values are means $\pm \mathrm{SD}(\mathrm{n}=8)$. ${ }^{*} P<0.05$ and ${ }^{* *} P<0.01$ versus FS group. 


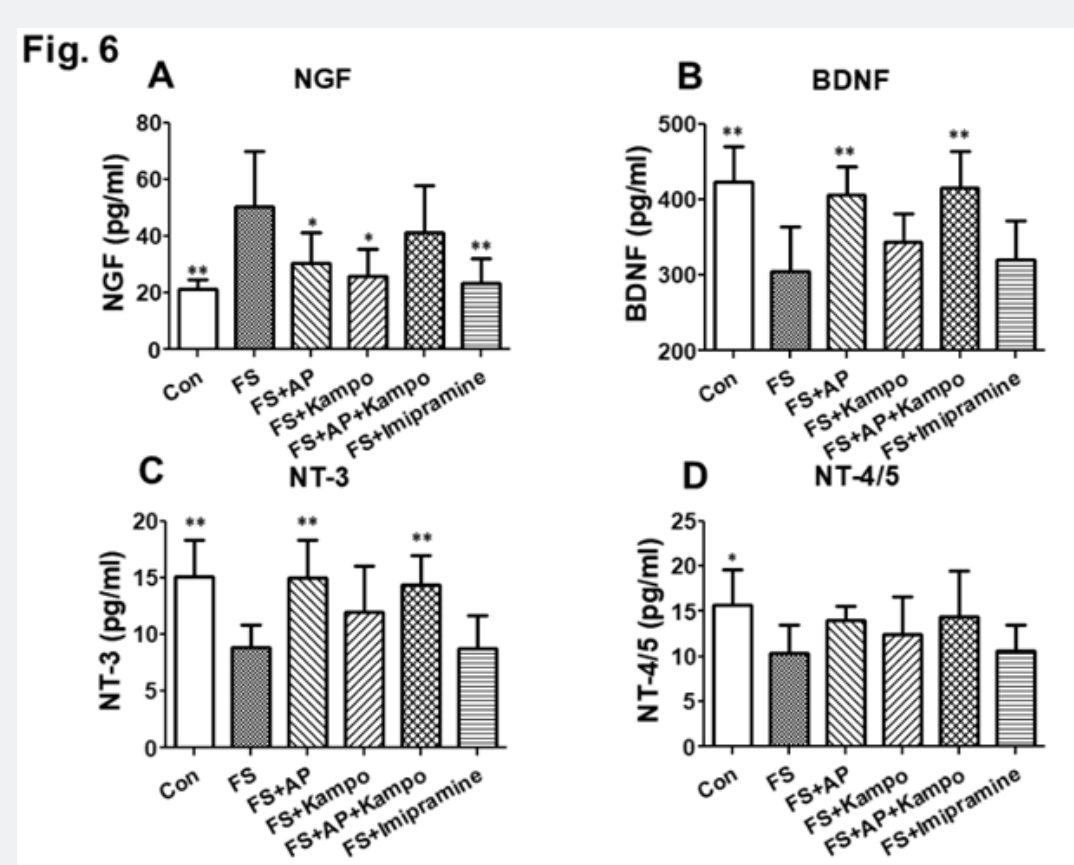

Figure 6: Expressions of NGF (A), BDNF (B), NT-3 (C), NT-4/5 (D) in the mice brain in the evaluation of therapeutic effect. Results are expressed as protein content measured by ELISA. Values are means $\pm \operatorname{SD}(n=8) .{ }^{\star} P<0.05$ and ${ }^{* \star} P<0.01$ versus FS group.

\section{Discussion}

The present study showed following results. The FS procedure made the immobility time of ICR mice significantly increased. In the therapeutic groups, the immobility time of AP, Kampo, combined, and imipramine groups was significantly lower than that of control group. Whereas, in the preventive groups, the immobility time of AP, Kampo, combined, and imipramine groups was not increased compared with control group. However, there was no significant difference in the immobility time between interventional groups in either the preventive group or the therapeutic group. Expressions of mRNA or protein of neurotrophic factors in the mice brains showed different patterns both in the preventive group and in the therapeutic group.

The method for producing ICR mouse in a state of depression by using forced swimming had been already established, and we found that this method was also useful in our investigation. In this study, the body weight of depressive mouse was as same as that before the forced swimming stress. It indicates that this method does not give ICR mice so much load. Judging from the results, we considered that this stress procedure could be allowed as a depression induction method.

Our investigation demonstrates that acupuncture is effective to depression according to the improvement of increased immobility time in the therapeutic groups, and the preservation of normal immobility time in the preventive groups. A large number of investigators have examined the state of depression in animal studies, many of which have found that chronic stress with a physical stressful stimulus could create a state of depression. Using animals in this state of depression, the effects of electroacupuncture have been examined at the "Bai-Hui" (GV 20) and "Yintáng" (Ex-HN3) points [3,22,27]. However, we showed that acupuncture without electric stimulation at the same acupoints had preventive and therapeutic effects on depression mice, following the report of the same effect on rats. This method of producing depression in mice seems to be very convenient and useful.

In addition, we found that acupuncture increased the decreased mRNA and protein expression of BDNF induced by the FS procedure in the therapeutic group, and that acupuncture maintain mRNA and protein expression of BDNF in spite of the FS procedure in the preventive group. Fluctuation in mRNA and protein expression of NT-3 was similar to that of BDNF. These findings suggest that acupuncture has preventive and therapeutic effect on depression through enhancing mRNA and protein expression of BDNF and NT-3.

We found that Kampo, the name of which is saikokaryukotsuboreito, significantly suppressed the onset of FS induced depression and successfully improved the prolonged immobility time of depression mice as well as imipramine. Expression pattern of mRNA and protein of neurotrophic factors in FS+Kampo group was similar to that in FS+Imipramine group in both the preventive and the therapeutic groups. These findings suggest that there might be difference in the mechanism to treat depression between acupuncture and Kampo and imipramine. Kampo medicine is Japanese traditional herbal medicine that is based on traditional Chinese medicine and has 
evolved to incorporate Japanese culture and proven scientific and medical knowledge [28]. Kampo products (mainly herbal extract formulations) were first included under the coverage of the Japanese National health insurance system in 1976. Today, we, Japanese doctors, are able to prescribe 148 kinds of Kampo formulas as well as the Western medicines within the National health insurance system. With less side effects of Kampo, we expect that Kampo will be utilized more frequently under the precise diagnosis.

Saikokaryukotsuboreito comprises of 10 kinds of crude drugs as described in the method. This formula is almost the same as shosaikoto with the addition of Ostreae testa, and Fossilia Ossis Mastodi. Shosaikoto works well for depressive condition. Ostreae testa, and Fossilia Ossis Mastodi are effective for tension from overactivity of the sympathetic nervous system.This function of saikokaryukotsuboreito seems to be effective for depression. In addition, it would be more effective, if accompanied by excessive muscle tension [29]. The characteristic of Kampo, saikokaryukotsuboreito might support that prolonged immobility time was significantly decreased in the therapeutic FS+Kampo group, and that immobility time was normally maintained in the preventive FS+Kampo group.

We did not observe that there were significant differences in the immobility time in either the preventive groups or the therapeutic groups, which were unexpected data, and unfortunately demonstrates that combination therapy of acupuncture and saikokaryukotsuboreito has no significant additive or synergistic effect for depression. However, protein expressions of NGF and NT-4/5 in FS+AP+Kampo group showed different patterns from FS+AP and FS+Kampo groups. These data suggest that there might be some difference in the mechanism for treating depression from acupuncture or Kampo monotherapy. That means the combined therapy has anti-depression effect through maintaining NGF and increasing NT-4/5. In the behavioral investigation, we unfortunately performed the evaluation of depression with only immobility time. We expect that we will be able to detect the severity of depression more precisely with more evaluation scales such as open-field test, hole-board test, elevated plus -maze test, light/dark test, and so on.

\section{Conclusion}

Our study indicates that the therapeutic effects of Acupuncture and Kampo Medicine for depression are through the regulation of neurotrophic factors including BDNF, which is different from imipramine. More importantly, there are different mechanisms in regulation of neurotrophic factors between Acupuncture monotherapy, Kampo monotherapy, and combined therapy with both of them.

\section{Acknowledgement}

This work was supported by JSPS KAKENHI Grant Number JP17K09332. The authors are grateful to Tsumura \& Co. for providing Kampo medicine.

\section{References}

1. World Health Organization (2010) mhGAP Intervention Guide for Mental, Neurological and Substance Use Disorders in Non-specialized Health Settings. Geneva: WHO: 1-107. ISBN: En 9789241548069.

2. Mishra S, Swain TR, Mohanty M (2013) Adverse drug reaction monitoring of antidepressants in the psychiatry outpatients department of a tertiary care teaching hospital. J Clin Diagn Res 7(6):1131-1134.

3. Smith CA, Armour M, Lee MS, Wang LQ Hay PJ (2018) Acupuncture for depression. Cochrane Database Syst Rev 4: 3.

4. Armour M, Smith CA, Wang LQ, Naidoo D, Yang GY, et al. (2019) Acupuncture treatment for depression-A systematic review and meta-analysis. J Clin Med 31: 8(8).

5. Zhn Y, Liu Q Zhuo LS (2009) Influence of electroacupuncture of "Baihui" (GV 20) and "Sanyinjiao" (SP 6) on hippocampal 5-HT and AChE immuno-activity in chronic depression rats. Zhen Ci Yan Jiu 34(1):1620.

6. Tanahashi N, Takagi K, Amagasu N, Wang G, Mizuno K, et al. (2016) Effect of acupuncture stimulation on rats with depression induced by water-immersion stress. Neuroscience Letters 618: 99-103.

7. Kamba S, Yamada K, Mizushima H, Asai M (1998) Use of herbal medicine for treating psychiatric disorders in Japan. Psychiatry and Clinical Neuroscience: 52(S6), Suppl, s331-s333.

8. Nishimura K, Plotnikoff GA, Watanabe K (2009) Kampo medicine as an integrative medicine in Japan. JMAJ 52(3): 147-149.

9. Kaptchuk TJ (2002) Acupuncture: theory efficacy, and practice, Ann. Intern. Med 136(5): 374-383.

10. Lacey JM, Tershakovec AM, Foster GD (2003) Acupuncture for the treatment of obesity: a review of the evidence, Int J Obes Relat Metab Disord 27(4): 419-427.

11. Nestler EJ, Barrot M, DiLeone RJ, Eisch AJ, Gold SJ et al. (2002) Neurobiology of depression, Neuron 34(1): 13-25.

12. Slattery DA1, Hudson AL, Nutt DJ (2004) Invited review: the evolution of antidepressant mechanisms, Fundam Clin Pharmacol. 18(1): 1-21.

13. Duman RS, Heninger GR, Nestler EJ (1997) A molecular and cellular theory of depression. Arch Gen Psychiatry 54(7): 597-606.

14. Duman RS (2009) Neuronal damage and protection in the pathophysiology and treatment of psychiatric illness: stress and depression. Dialogues Clin Neurosci 11(3): 239-255.

15. Shirayama Y, Chen AC, Nakagawa S, Russell DS, Duman RS (2002) Brain-derived neurotrophic factor produces antidepressant effects in behavioral models of depression. J Neurosci 22(8): 3251-3261.

16. Berman RM, Cappiello A, Anand A, Oren DA, Heninger GR, et al. (2000) Antidepressant effects of ketamine in depressed patients. Biol Psychiatry 47(4): 351-354.

17. Duman RS, Aghajanian GK (2012) Synaptic dysfunction in depression: potential therapeutic targets. Science 338(6103): 68-72.

18. Berton O, Nestler EJ (2006) New approaches to antidepressant drug discovery: beyond monoamines. Nat Rev Neurosci 7(2): 137-151.

19. Duman RS, Monteggia LM (2006) A neurotrophic model for stress-related mood disorders. Biol Psychiatry 59(12): 1116-1127.

20. Manji HK, Drevets WC, Charney DS (2001) The cellular neurobiology of depression. Nat Med 71(5): 541-547.

21. Porsolt RD, Le Pichon M, Jalfre M (1977) Depression: a new animal model sensitive to antidepressant treatments. Nature 266(5604): 730-732. 
22. Liu Q, Yu J, Mi WL, Mao-Ying QL, Yang R, et al. (2007) Electroacupuncture attenuates the decrease of hippocampal progenitor cell proliferation in the adult rats exposed to chronic unpredictable stress. Life Sci 81(21-22):1489-1495.

23. Takagi K, Tanahashi N, Amugasu N, Mizuno K, Kananokuchi J et al. (2017) Effect of manual acupuncture stimulation at "Bai-Hui" (GV 20) or "Yintáng" (Ex-HN3) on depressed rats. J Acupunct Meridian Stud 10(1): 26-32.

24. Adams SM, Miller KE, Zylstra RG (2008) Pharmacologic management of adult depression. Am Fam Physician 77(6): 785-792.

25. Bostwick JM (2010) A generalist's guide to treating patients with depression with an emphasis on using side effects to tailor antidepressant therapy. Mayo Clin Proc 85 (6): 538-550.

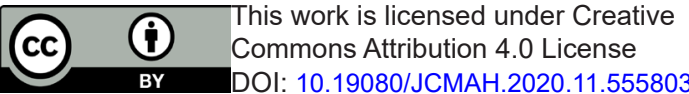

26. Mizoguchi K, Yuzurihara M, Ishige A, Aburada M, Tabira T (2003) Saikoka-ryukotsu-borei-to, a herbal medicine, ameliorates chronic stress-induced depressive state in rotarod performance. Pharmacol Biochem Behav 75(2): 419-425.

27. Smith CA, Hay PPJ, MacPherson H (2010) Acupuncture for depression. The Cochrane Library 1: 1-78. Wiley Online Library.

28. The Japan Society for Oriental Medicine (2005) Introduction to KAMPO Japanese Traditional Medicine. Elsevier Japan KK, Tokyo.

29. Nishimura K, Watanabe K, Toyoma $H$ (2009) Complete recovery from tension-type headache through Kampo medicine. J Altern Complemet Med 15(7): 799-801.

\section{Your next submission with Juniper Publishers will reach you the below assets}

- Quality Editorial service

- Swift Peer Review

- Reprints availability

- E-prints Service

- Manuscript Podcast for convenient understanding

- Global attainment for your research

- Manuscript accessibility in different formats ( Pdf, E-pub, Full Text, Audio)

- Unceasing customer service

Track the below URL for one-step submission https://juniperpublishers.com/online-submission.php 